\title{
Conductive Hearing Loss Induced by Experimental Middle-Ear Effusion in a Chinchilla Model Reveals Impaired Tympanic Membrane-Coupled Ossicular Chain Movement
}

\author{
Jennifer L. Thornton ${ }^{1,2}$ Keely M. Chevallier, ${ }^{1,3}$ Kanthaiah Koka, ${ }^{1}$ Sandra A. Gabbard, ${ }^{4}$ \\ AND DANiEl TOLLIN ${ }^{1,2,4}$ \\ ${ }^{1}$ Department of Physiology and Biophysics, University of Colorado School of Medicine, Anschutz Medical Campus, Aurora, CO, \\ USA \\ ${ }^{2}$ Neuroscience Training Program, University of Colorado School of Medicine, Aurora, CO, USA \\ ${ }^{3}$ MD Training Program, University of Colorado School of Medicine, Aurora, CO, USA \\ ${ }^{4}$ Department of Otolaryngology, University of Colorado School of Medicine, Aurora, CO, USA
}

Received: 27 June 2012; Accepted: 31 March 2013; Online publication: 25 April 2013

\begin{abstract}
Otitis media with effusion (OME) occurs when fluid collects in the middle-ear space behind the tympanic membrane (TM). As a result of this effusion, sounds can become attenuated by as much as $30-40 \mathrm{~dB}$, causing a conductive hearing loss (CHL). However, the exact mechanical cause of the hearing loss remains unclear. Possible causes can include altered compliance of the TM, inefficient movement of the ossicular chain, decreased compliance of the oval window-stapes footplate complex, or altered input to the oval and round window due to conduction of sound energy through middle-ear fluid. Here, we studied the contribution of TM motion and umbo velocity to a CHL caused by middle-ear effusion. Using the chinchilla as an animal model, umbo velocity $\left(V_{\mathrm{U}}\right)$ and cochlear microphonic $(\mathrm{CM})$ responses were measured simultaneously using sinusoidal tone pip stimuli $(125 \mathrm{~Hz}-$ $12 \mathrm{kHz}$ ) before and after filling the middle ear with different volumes $(0.5-2.0 \mathrm{~mL})$ of silicone oil (viscosity, 3.5 Poise). Concurrent increases in CM thresholds and decreases in umbo velocity were noted after the middle ear was filled with $1.0 \mathrm{~mL}$ or more of fluid. Across animals, completely filling the middle ear with fluid
\end{abstract}

Correspondence to: Daniel Tollin - Department of Physiology and Biophysics - University of Colorado School of Medicine - Anschutz Medical Campus, Aurora, CO, USA. Telephone: +1-303-7240625; fax: +1-303-7244501; email: Daniel.Tollin@ucdenver.edu caused 20-40-dB increases in CM thresholds and 15-35$\mathrm{dB}$ attenuations in umbo velocity. Clinic-standard 226-Hz tympanometry was insensitive to fluid-associated changes in CM thresholds until virtually the entire middle-ear cavity had been filled (approximately $>1.5 \mathrm{~mL}$ ). The changes in umbo velocity, CM thresholds, and tympanometry due to experimentally induced OME suggest CHL arises primarily as a result of impaired TM mobility and TM-coupled umbo motion plus additional mechanisms within the middle ear.

Keywords: conductive hearing loss, tympanometry, umbo velocity, middle-ear effusion, cochlear microphonic

\section{INTRODUCTION}

Otitis media with effusion (OME) is a type of middleear effusion (MEE) that results in an accumulation of fluid in the middle-ear space (Bluestone and Klein 1995). In children, OME is the most frequent cause of hearing loss, with $\sim 90 \%$ of children experiencing an episode of OME before the age of 2 (Paradise et al. 1997). MEEs can result in a mild to moderate conductive hearing loss (CHL), a reversible hearing loss in which acoustic input to the inner ear is attenuated. In some cases, MEEs can attenuate sound by more than $30 \mathrm{~dB}$ (Kokko 1974; Bluestone et al. 
1973). Previous studies have shown that the amount of fluid in the middle ear can significantly impact the magnitude of CHL, regardless of the viscosity of the fluid (Brown et al. 1983; Wiederhold et al. 1980; Jeselsohn et al. 2005; Ravicz et al. 2004; Thornton et al. 2012). Furthermore, CHLs caused by MEEs have been shown to alter the binaural cues to sound location as represented by the inputs to the inner ear, the interaural time and level differences (Hartley and Moore 2003; Thornton et al. 2012).

Previous studies have shown that children with chronic MEEs have difficulty with binaural processing tasks later in life, even after the cause of the CHL (e.g., drainage of middle-ear fluid) has been corrected and the peripheral auditory system (audiograms, hair cell thresholds, etc.) has returned to normal (Hall and Derlacki 1986; Pillsbury et al. 1991; Moore et al. 1991, 2003; Hogan and Moore 2003; Hall et al. 1998). Since long-term behavioral complications can arise from chronic CHL due to MEEs during development, early clinical identification and intervention in ameliorating MEEs is critical. However, MEEs can present without the painful symptoms that often coincide with infection, making it difficult for parents of infants and their pediatricians to recognize the presence of MEEs at all (for review see Whitton and Polley 2011). Additionally, it is entirely possible for children to have an MEE without a corresponding CHL. Thus while being clinically diagnosed as having OME, these children would not be expected to experience any long-term hearing or binaural processing deficits (Whitton and Polley 2011). In other words, it is not the OME per se that causes long-term and persistent hearing problems but rather the CHL that may or may not result as a consequence.

In the clinic, while it is relatively easy to diagnose OME, it is much more difficult to determine the magnitude of a CHL using audiometry or evoked potentials. Tympanometry, a method used to measure the physical properties of the middle-ear system and tympanic membrane (TM) mobility, is commonly used clinically to diagnose the presence of MEEs (Hall and Chandler 1994). However, it has been shown that the use of clinic-standard $226 \mathrm{~Hz}$ tympanometry is not always sensitive enough to detect the presence of middle-ear fluid, particularly in infants and small children (Orchik et al. 1978; Wiederhold et al. 1980; Margolis et al. 1995). As tympanometry is designed to test the compliance of the TM and quantify middle-ear pressure, it does not provide much direct information about the resultant movement of the ossicular chain (i.e., middle-ear bones) that provides the mechanical input to the inner ear. Other acoustic response tests using admittance-reflectance measurements can more accurately diagnose middle-ear effusions that result in a CHL.
However, they still cannot provide much information about the movement of the ossicular chain and how that might be affecting the inputs to the inner ear.

By measuring umbo velocity $\left(V_{\mathrm{U}}\right)$ in the presence of an MEE, we can test the hypothesis that ossicular chain movement is impaired when fluid is present in the middle-ear space, thus causing a CHL. Recently, Ravicz et al. (2004) quantified in human cadaveric temporal bones the change in the umbo velocity transfer function as a result of an increasing volume of middle-ear fluid, but they did not test the more proximal aspects of the middle-ear system such as the resultant inputs to the inner ear. If the CHL is partially caused or modified by a mechanism proximal to the umbo, these experiments would not be able to accurately assess that change, as TM and umbo velocity measurements do not provide data on the transmission of sound through the middle ear. While changes in $V_{\mathrm{U}}$ must certainly contribute substantially to the CHL due to MEE, other measurements, such as stapes footplate velocity or cochlear microphonics (CMs) produced by the outer hair cells of the inner ear, must be used to identify any contributing or modifying factors to the CHL by the remainder of the middle-ear system.

For the experiments in this study, the chinchilla (Chinchilla lanigera) was used due to its "humanlike" auditory system. The chinchilla has a comparable audiogram to that of humans (hearing range of $\sim 50 \mathrm{~Hz}-33 \mathrm{kHz}$ ) in addition to similar physiological and anatomical characteristics (tympanic membrane size, cochlea size, etc.) as compared to the human auditory system (Miller 1970; Vrettakos et al. 1988; Heffner and Heffner 1991; Niemiec et al. 1992). These features make the chinchilla a proper animal model for comparisons to human studies.

The main goal of this study was to test the hypothesis that an MEE-induced CHL as evidenced at the level of the inner ear (cochlea) by CM measurements can be accounted for by changes in tympanic membrane and ossicular chain movement. As tympanometry is one of the main diagnostic tests used in clinical settings as a means to diagnose CHL, we also test the hypothesis that tympanometry measurements will be indicative of an MEE-induced CHL. This hypothesis is based on the premise that because changes in the compliance of the TM should be observed in the presence of a CHL due to MEE, tympanograms should reflect the altered mobility of the TM. To test these hypotheses comprehensively, we instilled different volumes of fluid into the middle-ear space of adult chinchillas to simulate an MEE-induced CHL. After each fluid instillation, we measured the movement of the TM (via tympanometry and $V_{\mathrm{U}}$ measurements conducted in separate animals) as well 
as the outer hair cell response (via CM measurements) in response to sound to see how these responses jointly changed as a result of fluid in the middle-ear space.

\section{METHODS}

\section{Animal preparation}

All experiments and methods were approved by the University of Colorado School of Medicine Institutional Animal Care and Use Committee. Nine ears were studied in five adult chinchillas (C. lanigera; three females, two males) weighing 0.60 to $0.82 \mathrm{~kg}$ (mean, $0.67 \mathrm{~kg} ; \mathrm{SD}, 100 \mathrm{~g}$ ). The animals were anesthetized with an initial intramuscular dose of ketamine hydrochloride (KetaVed; $30 \mathrm{mg} / \mathrm{kg}$ ) and xylazine hydrochloride (TranquiVed; $3.75 \mathrm{mg} / \mathrm{kg}$ ). Anesthesia was maintained with supplemental doses of ketamine $(15 \mathrm{mg} / \mathrm{kg}$, i.m.) and xylazine $(2.5 \mathrm{mg} /$ $\mathrm{kg}$, i.m.) based on reflex response to a paw pinch and changes in physiologic parameters (heart rate, etc.). Each animal underwent tracheotomy tube placement to facilitate ventilation. The animal's head was secured in a custom-designed bite bar. Heart rate, blood oxygen levels $\left(\mathrm{SpO}_{2}\right)$, respiratory rate, and end-tidal $\mathrm{CO}_{2}$ were measured continuously via a capnograph (SurgiVet V90040, Waukesha, WI, USA). Body temperature was continuously monitored with a surface temperature probe placed underneath the animal and maintained with a heating pad at $36{ }^{\circ} \mathrm{C}$ (model TC 100; CWE, Inc., Ardmore, PA, USA).

A midline incision was made from between the eyes to the inferior portion of the skull. The skin and fascia were bluntly dissected off of the bullae. The pinnae were removed bilaterally down to the bony portion of the external auditory canal. In order to model an MEE, a $6-7-\mathrm{mm}$ diameter hole was drilled into the posterior aspect of the bullae on both sides using a dental drill. A polythene tube (inner diameter, 0.125 in.) was inserted into these posterior bullae holes bilaterally and sealed firmly in place with dental acrylic and superglue. A small vent hole $(\sim 2-3 \mathrm{~mm}$ in diameter) was drilled into the superior portion of the bulla bilaterally to equalize middle-ear pressure. No additional tubing was attached to the vent hole.

A 3-mL syringe containing silicone oil was fitted with a 14-gauge needle, and the needle was inserted into the opening of the polythene tube attached to the posterior bulla. Silicone oil (polydimethylsiloxane fluid, Sigma-Aldrich Co. Ltd., St. Louis, MO) of a viscosity of 3.5 Poise (approximately the viscosity of fingernail polish) was used to implement the experimental model of an MEE, and was injected through the polythene tube at a slow and constant rate to ensure accurate measurements. This viscosity was recently shown to produce reliable mild-moderate CHL ( 20-30 dB) in our animal model (Thornton et al. 2012). Silicone oil has a low electrical conductance, and therefore is not expected to interfere with $\mathrm{CM}$ measurements. The filling tubes, syringes, and needle were put into place before the baseline CM or tympanometry measurements were taken. After each silicone oil injection, the polythene tube was clamped at the end to prevent the silicone oil from leaking out of the tube.

Cochlear microphonic measurements and acoustic stimulus presentation

For CM measurements, a small (2-3-mm diameter) hole was bored through the bony wall of each bulla posteriorly to allow access to the round window niche. Teflon-insulated, silver electrodes (bare wire diameter, 0.005 in.) were advanced through the hole by hand and placed at the round window niche. Placement of the electrode was confirmed via an oscilloscope. The electrode was fixed in place and the hole in the bulla was completely closed and sealed using dental acrylic. The differential electrode was placed on the neck musculature while the ground electrode was placed on the paw.

Free-field, calibrated sinusoidal stimuli were presented for CM measurements. Sinusoids (pure tones; five frequencies per octave from 0.25 to $12 \mathrm{kHz})$ had a total duration of $10 \mathrm{~ms}(2.5 \mathrm{~ms}$ rise/ fall phase and a 5-ms plateau) with a 40-ms interstimulus period. Stimuli were presented approximately 12 in. directly in front of the animal via a loudspeaker (TDT model FF1, Alachua, FL). Speaker placement regarding the TM did not change during the experiment, as the animal and speaker were held in place by a stereotaxic apparatus and loudspeaker mounting bracket, respectively. Because sounds were delivered via free-field stimuli presentation, changes in middle ear admittance due to fluid accumulation in the middle ear were not an issue in this study. The sound delivery system was calibrated for tones between 0.1 and $20 \mathrm{kHz}$ in $100 \mathrm{~Hz}$ steps via a 256-tap linear phase finite impulse response filter implemented in real time by a TDT RX6 (Alachua, FL) that provided a virtually flat acoustic response with a maximum output of $~ 90-95 \mathrm{~dB}$ SPL at the TM for frequencies $<20 \mathrm{kHz}$. For calibration, the tip of a probe tube calibration microphone (Bruel and Kjaer, Type-4182) was placed in the external ear canal $\sim 2 \mathrm{~mm}$ from the TM. The CM signals were differentially amplified $(\times 1,000$, DAM ISO-50 or DAM ISO- 80 , WPI, Sarasota, FL), filtered $(10-20,000 \mathrm{~Hz})$ and verified by oscilloscope. All stimulus presentation, acquisition, and processing were done using custom written software in MATLAB®. This process was done 
for both ears. CM measurements were conducted simultaneously with $V_{\mathrm{U}}$ measurements while varying the sound stimulus level from -20 to $100 \mathrm{~dB}$ SPL. We were concerned that the vent hole in the bulla might cause either a gain or attenuation of sound unrelated to the presence of fluid, so all baseline condition measurements included the vent hole so comparison measurements excluded these effects. We found that a small hole in the bulla does not affect how much sound is entering the middle-ear space, as evidenced via $\mathrm{CM}$ threshold and umbo velocity measurements. However, such a hole in the bulla can change the compliance of the system, as described in more detail in the "Discussion" section.

\section{Umbo velocity measurements}

In order to visualize the TM and the tip of the umbo, the lateral wall of the bony external auditory canal was removed until the umbo tip was easily visible from the lateral view. With the aid of a surgical microscope, reflective beads (Scotchlite ${ }^{\mathrm{TM}}, 3 \mathrm{M}, \mathrm{MN}$, USA) were carefully placed on the tip of the umbo to increase the laser Doppler vibrometer (LDV) signal-to-noise ratio. A Polytec Hearing Laser Vibrometer (HLV1000; Polytec, Waldbronn, Germany) was fixed to a Zeiss standing microscope, and the laser beam from the HLV head was aimed through the microscope via a joystick. The aiming prism was positioned underneath the microscope objective, and the laser reflection was viewed via the microscope. Both the microscope and the LDV were fixed to a surgical table in the middle of a vibration isolation soundproof chamber. The umbo velocity $\left(V_{\mathrm{U}}\right.$, millimeter per second) was recorded simultaneously with and using the same stimuli as the CM measurements. The laser was pointed normal to the plane of the TM and umbo so no correction for laser angle was necessary.

Baseline CM and umbo velocity measurements were taken in each animal before any silicone oil was instilled into the middle-ear space. After the baseline measurements, $0.50 \mathrm{~mL}$ of silicone oil was very slowly injected into the middle-ear space. The polythene tubing (see above) of known volume was pre-filled with silicone oil so that the desired volumes of oil (i.e., $0.5,0.75 \mathrm{~mL}$ ) could be accurately delivered into the bullae. Moreover, as the tip of the injection needle was flush with the end of the filling tube as it was connected to the bullae, errors in the estimation of the injected fluid volume were expected to be negligible. Fluid injection into the bullae was jointly facilitated by very slow injection rates and by allowing air to vent through the superior vent hole in the bulla. Additional silicone oil was injected into the bulla in $0.25 \mathrm{~mL}$ increments, with cochlear microphonic and umbo velocity measurements taken after each addi- tion of fluid. Experiments were conducted with 0.5-, $0.75-, 1.0-, 1.25-$, and $1.5-\mathrm{mL}$ volumes of silicone oil and the last measurement taken with the bulla completely filled with silicone oil as evidenced by oil just beginning to come out of the superior vent hole $(\sim 2.0 \mathrm{~mL})$. This method of MEE simulation was adapted, in part, from the methods used by Goodhill and Holcomb (1958) and Hartley and Moore (2003). One viscosity of silicone oil was used (as opposed to a variety of viscosities) based on previous research that suggests that attenuation of sound as a result of middle-ear fluid depends mostly on fluid volume and not viscosity (Brown et al. 1983; Wiederhold et al. 1980; Marsh et al. 1985; Ravicz et al. 2004; Jeselsohn et al. 2005; Thornton et al. 2012). As a confirmation that the bullae were completely filled with fluid via this method, in some animals the silicone oil was mixed with food coloring. Based on visual inspection, the percentage of the TM in contact with fluid was recorded for each fluid volume (Fig. 1). The chinchilla middle-ear space is comprised of several different spaces, some of which are quite small (Browning and Granich 1978). Thus, it is unlikely that the fluid in the middle-ear space filled all of these cavities. However, the dye staining estimates that were used confirmed that the large air space immediately behind the TM was indeed filled with fluid.

\section{Tympanometry measurements}

For tympanometry measurements, a standard clinical middle-ear analyzer (GSI TympStar, Eden Prairie, $\mathrm{MN}$ ) was used to deliver a probe tone via a probe tube eartip (11-mm diameter), which was held tightly in place manually over the bony portion of the

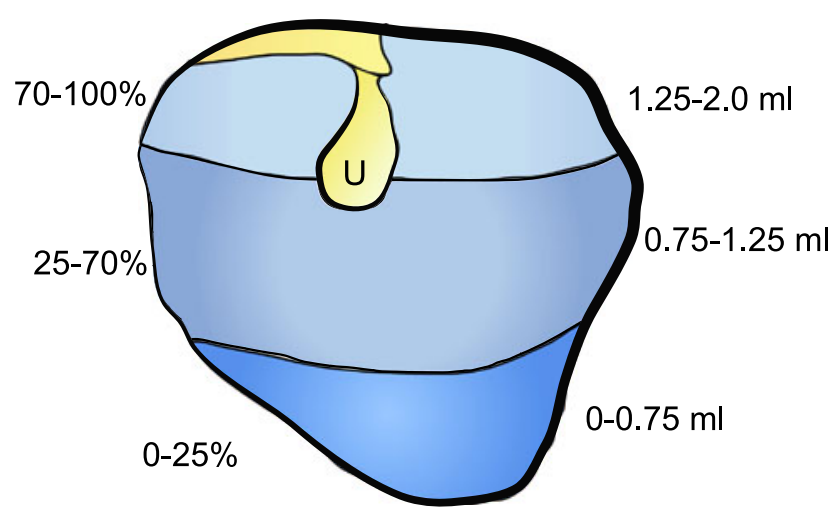

FIG. 1. Observed fluid levels at the tympanic membrane. Silicone oil was mixed with food coloring to visualize the amount of TM that was covered by fluid during the fluid instillation process. With $0.75 \mathrm{~mL}$ of silicone oil in the middle-ear space, $25 \%$ of the overall TM area was covered with fluid. With $1.0 \mathrm{~mL}$ of silicone oil, the TM was $\sim 70 \%$ covered, and at $1.25 \mathrm{~mL}$ of silicone oil in the middle ear, the TM was $100 \%$ covered by fluid. The location of the umbo is indicated by the letter $U$. 
external auditory canal. Margolis et al. (1995) used a similar method in their study of MEE in chinchilla. An airtight seal between the probe tip and the ear canal was maintained throughout all tympanometric tests. A tympanometric measurement cannot be obtained without a tight seal, a fact that allowed for rapid confirmation that an acceptable measurement was being made. Due to this fact, tympanometric measurements could not be made simultaneously with CM and umbo velocity measurements. Baseline diagnostic tympanometry measurements were taken using an audiology clinic-standard $226 \mathrm{~Hz}$ probe frequency prior to the introduction of fluid. In addition to $226 \mathrm{~Hz}$ tympanometry, tympanograms were also recorded for 500-, 700-, 1,100-, 1,400-, 1,600-, 1,800-, and $2,000-\mathrm{Hz}$ probe frequencies, with a pressure sweep at $200 \mathrm{daPa}(1 \mathrm{daPa}=10 \mathrm{~Pa})$. Tympanograms were classified based on the Jerger classification scheme (Jerger 1970). Additionally, the morphology of the peaks for each frequency was compared between the baseline condition and each fluid level when tympanometry curves did not conform to Jerger standards. Tympanometry measurements were not compensated for the effect of the ear canal between the probe and the eardrum, and any effects of fluid accumulation in the middle ear on pressure measured in front of the TM were not controlled for.

\section{RESULTS}

Cochlear microphonic thresholds increase as fluid volume in the middle ear increases

Changes in cochlear microphonic (CM) thresholds were measured after each instillation of silicone oil (as well as at baseline) at low $(0.25-1 \mathrm{kHz})$, medium (1$3 \mathrm{kHz}$ ), and high (3-8 kHz) frequencies (Fig. 2A, B). The CM is a sound-evoked electrophysiological potential generated by the peripheral auditory system representing the ensemble activity of the outer hair cells. The CM reproduces acoustic waves over a wide range of intensities; as such, the CM provides an electrophysiological proxy of the effective vibratory energy that reaches the inner ear (Dallos 1973). Figure 2A shows the across animal mean ( $n=5$ ears $) \pm$ $\mathrm{SD}$ for $\mathrm{CM}$ thresholds as a function of sound frequency. Baseline measurements are not shown as they are not significantly different from 0.5 -mL fluid instillation values for CM thresholds. CM thresholds were increased by the greatest amount with $1.25 \mathrm{~mL}$ of fluid in the middle-ear space. We also wanted to determine if there was a frequency effect on the amount of sound attenuation (measured via $\mathrm{CM}$ thresholds) caused by middle-ear fluid (Fig. 2B). While Figure 2A shows CM thresholds, Figure 2B shows the amount of attenuation in decibels relative to the baseline measurements for the
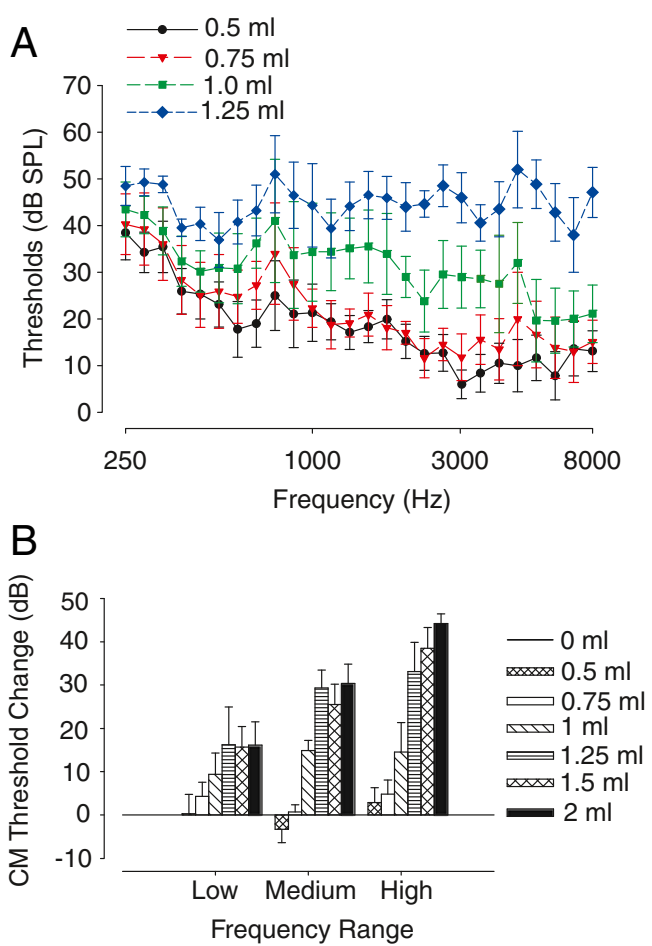

FIG. 2. CM absolute thresholds and threshold changes as a result of middle-ear fluid volume. A Across-animal ( $n=5$ ears) mean CM threshold as a function of frequency; error bars indicate $\pm 1 \mathrm{SD}$. As the volume of middle-ear fluid was increased from $0.5 \mathrm{~mL}$, the $\mathrm{CM}$ thresholds increased. B The data in panel A were further analyzed by computing the MEEinduced change in $\mathrm{CM}$ thresholds relative to the baseline with no middleear fluid in three frequency ranges: low $(0.25-1 \mathrm{kHz})$, medium $(1-3 \mathrm{kHz})$, and high $(3-8 \mathrm{kHz})$. With 0.5 and $0.75 \mathrm{~mL}$ of fluid in the middle ear, there was little change in the CM thresholds. For fluid volumes greater than $1.0 \mathrm{~mL}$, there were substantial increases in $\mathrm{CM}$ thresholds in all frequency ranges, indicating a $\mathrm{CHL}$. In the low-frequency range, the maximum change in $\mathrm{CM}$ thresholds $(18 \mathrm{~dB})$ was reached by $1.0 \mathrm{~mL}$ of fluid in the middle-ear space. Large increases in CM thresholds for medium- and high-frequency sounds were observed when $1.0 \mathrm{~mL}$ of fluid was present in the middle-ear space, with near maximum threshold increases occurring when volumes of $1.25 \mathrm{~mL}$ or more of fluid were present (100\% TM area-fluid contact). Maximum threshold increases for medium- and high-frequency ranges were 30 and $42 \mathrm{~dB}$, respectively. Baseline measurements are not shown as they are not significantly different from $0.5-\mathrm{mL}$ fluid instillation values for $\mathrm{CM}$ thresholds.

low, medium, and high-frequency ranges (as defined above and in the figure caption). For the low-frequency range of stimuli, fluid volume had a significant effect on CM thresholds (single-factor ANOVA, $F_{1,6}=33.05, p<$ 0.01). Similar findings were observed for medium (single-factor ANOVA, $F_{1,6}=153.02, p<0.01$ ) and high frequencies (single-factor ANOVA, $F_{1,6}=126.24, p<$ $0.01)$. A post hoc Sheffe test revealed no significant effects $(p>0.05)$ of fluid level on CM thresholds for all frequencies after more than $1.0 \mathrm{~mL}$ of fluid was instilled in the middle-ear space; that is, fluid levels of 1.25, 1.5, and $2.0 \mathrm{~mL}$ produced similar results. The maximum increase in CM thresholds compared to baseline measurements was frequency-dependent, with increases 
of 18,28 , and $44 \mathrm{~dB}$ at low, medium, and high frequencies, respectively.

The area of $\mathrm{TM}$ in contact with the fluid increased as more fluid was instilled in the middle-ear space. When $0.75 \mathrm{~mL}$ of 3.5 Poise silicone oil was instilled in the middle-ear space, the area of the TM in contact with the fluid was visually estimated to be $\sim 25 \%$ of the total TM area (Fig. 1, $n=5$ ears). As the TM is translucent and nearly transparent, the amount of silicone oil in the middle ear could be visualized easily. With $1.0 \mathrm{~mL}$ of fluid present in the middle ear, fluid contact with the TM increased to $50-70 \%$ coverage $(n=5$ ears). The TM was completely covered with $1.25 \mathrm{~mL}$ of fluid in the middle ear in all ears studied. Across animals and frequency ranges (low, medium, high), when $0-25 \%$ of the medial TM area was contacted by fluid $(0-0.75 \mathrm{~mL})$, the $\mathrm{CM}$ thresholds did not show a significant difference compared to baseline CM thresholds (Paired $t$ test, $\left.t_{4}=-1.0, p=0.33\right)$.

Tympanometry measurements with fluid in the middle-ear space

After establishing the amount of sound attenuation that is present at the level of the inner ear as a result of MEE-induced CHL, we then used tympanometry measurements to examine how the middle ear responded to acute CHL. Using a standard 226- $\mathrm{Hz}$ probe tone, $75 \%$ of ears tested exhibited a type A tympanogram in both the baseline and initial fluid (0.5 mL) conditions (Fig. 3). Type A tympanograms are characterized by a sharp and narrow peak that is centered around $0 \mathrm{daPa}$, or ambient pressure. Type A tympanograms indicate normal pressure within the middle ear and may indicate normal sound transmission capabilities of the middle-ear system (Jerger 1970). Additional fluid instillation to a total volume of $1.0 \mathrm{~mL}$ revealed noticeable shortening and widening of the tympanometry curves (Fig. 3). Type B tympanograms, characterized by a flat curve with no discernible peak, were not observed until the bulla had been almost completely filled with at least $1.5 \mathrm{~mL}$ of fluid and the TM was completely covered. Type B tympanograms are indicative of fluid in the middleear space or TM perforation (Hall and Chandler 1994). Clinically, the presence of a type B tympanogram confirms the presence of otitis media with effusion.

Currently, $226 \mathrm{~Hz}$ is the standard frequency used in audiology clinics to examine the different properties of the TM as well as to confirm the presence of fluid in the middle-ear space for adults and children over 6 months of age. Some studies have suggested that $226 \mathrm{~Hz}$ may not be sensitive enough to detect MEE in infants and young children who have middle-ear volumes that are much smaller than those of adults (Holte et al. 1991; Keith 1975; Himmelfarb et al. 1979; Margolis and Popelka 1975) and clinical protocols for children less than 6 months of age currently recommend using a $1,000-\mathrm{Hz}$ probe tone (JCIH 2007). To further examine this issue, we used multi-frequency tympanometry (MFT) to determine if there was a more optimal probe frequency for detecting the presence of fluid in the middle ear. Consistent with Piskorski et al. (1999), we found that the use of higher probe frequencies $(1,400-1,800 \mathrm{~Hz})$ was more sensitive than $226 \mathrm{~Hz}$ for detecting the presence of fluid in the middle-ear space $(n=6$, Table 1$)$. With $1.0 \mathrm{~mL}$ of 3.5 Poise silicone oil in the middle ear, 1,400 $\mathrm{Hz}$ tympanometry resulted in a type $\mathrm{B}$ tympanogram in $\sim 67 \%$ of ears tested (Table 1 ; Fig. 4). In contrast, $226 \mathrm{~Hz}$ tympanometry did not yield a type B tympanogram with $1.0 \mathrm{~mL}$ of fluid in the middle ear for all ears tested (Table 1), even though a majority of the TM $(\sim 70 \%)$ was covered by fluid.

Umbo velocity decreases with increased levels of fluid in the middle-ear space

Tympanometry relies on detecting altered movement of the TM as caused by an MEE. The CM results show that fluid in the middle ear is attenuating sound, but 226-Hz tympanometry results do not indicate abnormal, type B tympanograms for most fluid levels. By measuring umbo velocity, it might be possible to reconcile the fact that changes seen at the level of the inner ear are not seen at the TM.

The change in umbo velocity $\left(V_{\mathrm{U}}\right)$ was recorded for each additional fluid instillation and compared to the baseline condition. As the laser was oriented normal to the plane of the TM and umbo, it was not necessary to correct $V_{\mathrm{U}}$ for laser angle. We compared the $V_{\mathrm{U}}$ measurements in three separate groups based upon the same stimulus frequency groups as the CM measurements: low- $(0.25-1 \mathrm{kHz})$, medium- (1$3 \mathrm{kHz}$ ), and high-frequencies (3-8 kHz; Fig. 5). Changes in the magnitude of the umbo velocity were converted to decibels.

Figure 5A shows the across-animal mean of umbo velocity measurements for different fluid levels and frequencies. Umbo velocities were decreased by the greatest amount with $1.25 \mathrm{~mL}$ of fluid in the middleear space. Figure 5B separates the data into different frequency ranges to examine the effect that frequency might have on umbo velocity. Baseline measurements are not shown as they are not significantly different from $0.5 \mathrm{~mL}$ fluid instillation values for umbo velocity measurements. Changes in $V_{\mathrm{U}}$ were frequency dependent, with the largest changes occurring at the highest frequencies tested. With $100 \%$ of the TM covered with fluid, $V_{\mathrm{U}}$ decreased by $\sim 11 \mathrm{~dB}$ (mean $=11.6 \mathrm{~dB}$, 

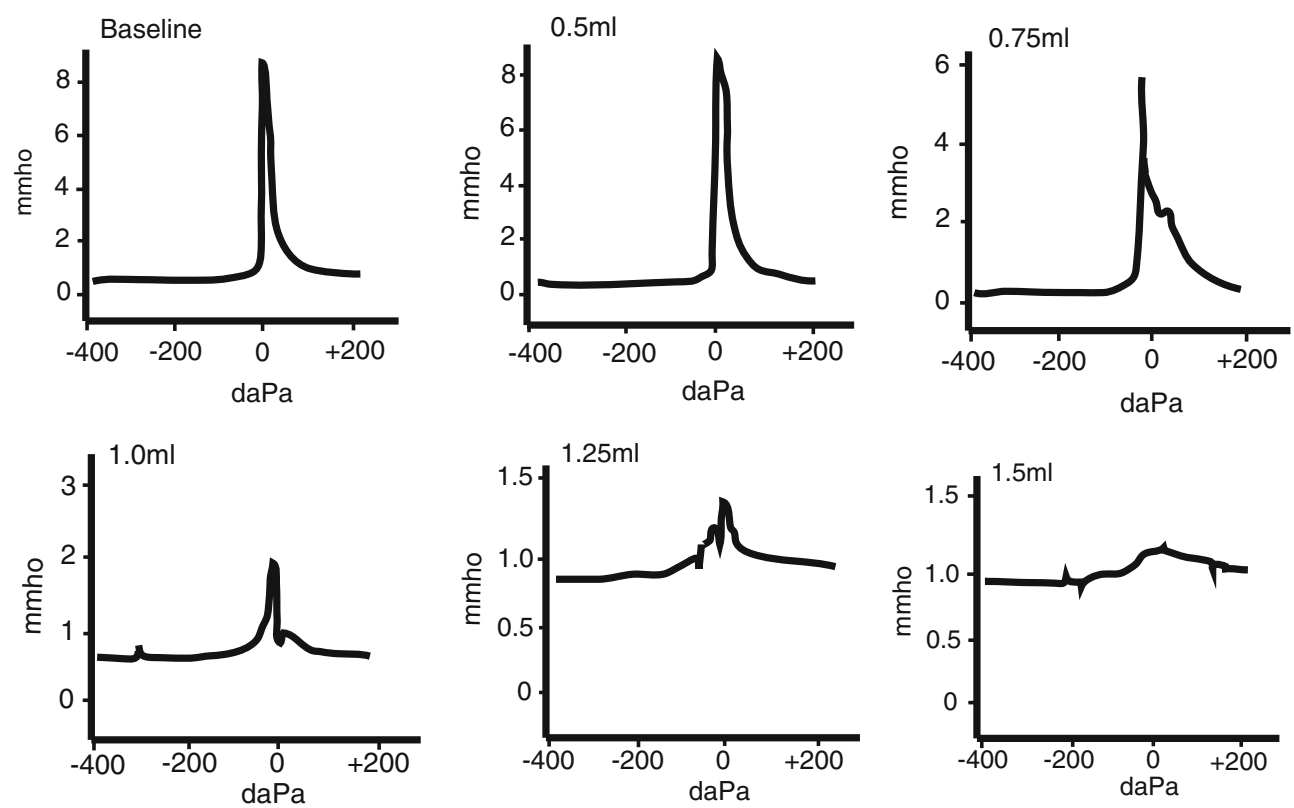

FIG. 3. The 226-Hz clinic-standard tympanometry with various volumes of silicone oil in the middle ear. The $226-\mathrm{Hz}$ tympanometry did not yield type B tympanograms consistent with MEEs until the middle-ear space was nearly completely filled with fluid $(1.5 \mathrm{~mL})$. With $0.75 \mathrm{~mL}$ of fluid in the middle ear, at least $25 \%$ of the TM is in contact with the fluid (Fig. 1).

$\mathrm{SD}=0.2$ ) at low frequencies, $\sim 24 \mathrm{~dB}$ (mean $=24.3, \mathrm{SD}=$ 0.87 ) at medium frequencies, and $\sim 33 \mathrm{~dB}$ (mean $=$ 33.8, $\mathrm{SD}=1.53$ ) at the highest frequencies (Fig. 5A, B). Previous results have also shown that $V_{\mathrm{U}}$ is attenuated by MEE more at higher stimulus frequencies than lower (Ravicz et al. 2004). We also found that $V_{\mathrm{U}}$ was influenced by the amount of TM that was covered by fluid. For example, for low-frequency sounds, there was a significant effect of the volume of silicone oil in the middle ear on $V_{\mathrm{U}}$ (single-factor ANOVA, $F_{1,6}=$ 23.1, $p<0.01, n=5$ ). A post hoc Sheffe test revealed no significant effect of fluid on $V_{\mathrm{U}}$ for fluid volumes of $0.75 \mathrm{~mL}$ or greater $(p>0.05)$; that is, once at least $0.75 \mathrm{~mL}$ of fluid was in the middle ear, additional fluid amounts did not produce additional attenuation of $V_{\mathrm{U}}$. The maximum recorded $V_{\mathrm{U}}$ attenuation for the low-frequency stimulus range was $16 \mathrm{~dB}$. A similar pattern of the effects of fluid volume was found for
Some widening of the tympanometry curves is observed, but the tympanometry curve still resembles a type A tympanogram. At $1.25 \mathrm{~mL}$ of fluid in the middle ear (100\% TM area covered), the tympanometry curve still retains a small peak around 0 daPa.

medium frequency (single-factor ANOVA, $F_{1,6}=$ $164.75, p<0.01$ ) and high-frequency sounds (singlefactor ANOVA, $F_{1,6}=314.9, p<0.01$ ), in that there was no significant difference after the majority of the TM was covered by fluid. Additionally, post hoc Sheffe tests revealed no significant effect $(p>0.05)$ of fluid volume on $V_{\mathrm{U}}$ when fluid volume was $1.25 \mathrm{~mL}$ or more for both medium- and high-frequency sounds. The maximum recorded $V_{\mathrm{U}}$ attenuations were 28 and $44 \mathrm{~dB}$ for medium- and high-frequency sounds, respectively.

\section{Relationship between $V_{U}$ and $\mathrm{CM}$}

Both $V_{\mathrm{U}}$ and $\mathrm{CM}$ thresholds were dependent on stimulus frequency, the volume of fluid in the middle-ear space, and the percentage of the TM area that was contacted by fluid. In Figure 6 we compared

TABLE 1

Percentage of type B tympanograms present across different fluid levels and probe tone frequencies $(n=6)$

\begin{tabular}{|c|c|c|c|c|c|c|c|c|}
\hline & $226 \mathrm{~Hz}$ & $500 \mathrm{~Hz}$ & $700 \mathrm{~Hz}$ & $1,100 \mathrm{~Hz}$ & $1,400 \mathrm{~Hz}$ & $1,600 \mathrm{~Hz}$ & $1,800 \mathrm{~Hz}$ & $2,000 \mathrm{~Hz}$ \\
\hline Baseline & - & - & - & - & - & - & - & - \\
\hline $0.5 \mathrm{~mL}$ & - & - & - & - & - & - & - & - \\
\hline $0.75 \mathrm{~mL}$ & - & - & - & - & 50 & & 50 & 50 \\
\hline $1.0 \mathrm{~mL}$ & - & - & - & 33.3 & 66.6 & 50 & 100 & 100 \\
\hline $1.25 \mathrm{~mL}$ & - & - & 50 & 100 & 100 & 100 & 100 & 100 \\
\hline $1.50 \mathrm{~mL}$ & 100 & 50 & 50 & 100 & 100 & 100 & 100 & 100 \\
\hline
\end{tabular}

Using a 226- $\mathrm{Hz}$ probe tone, type B tympanograms are only observed after the middle-ear space has been filled with $1.5 \mathrm{~mL}$ of fluid. In contrast, higher probe tone frequencies $(>1,400 \mathrm{~Hz})$ can detect type B tympanograms with $0.75 \mathrm{~mL}$ of fluid in the middle ear 



FIG. 4. High-frequency tympanometry $(1,400 \mathrm{~Hz})$ with various volumes of silicone oil in the middle ear. Type B tympanograms were more common when using a $1,400-\mathrm{Hz}$ probe tone for tympanometry measurements. At $1,400 \mathrm{~Hz}$, subtle changes in the tympanometry curve width were noticeable even with the first $0.5 \mathrm{~mL}$ of added middle-ear fluid. With $0.75 \mathrm{~mL}$ of fluid in the middle ear, the curves were noticeably shorter and wider. Type B tympanograms were seen at $1.0 \mathrm{~mL}$ of fluid in the middle ear. Across all animals, fluid levels above $1.0 \mathrm{~mL}$ resulted in type B tympanograms when using high-frequency probes. the MEE-induced changes in CM thresholds to the corresponding reductions in $V_{\mathrm{U}}$ measurements in all animals for all fluid volume levels and for all frequencies comprising each of the three frequency ranges. The linear regression of the data comprising each of the three frequency ranges indicates a strong positive correlation ( $r$ values of $0.73,0.88$ and 0.86 for the low $(n=385)$, medium $(n=280)$, and high $(n=245)$ frequency ranges, respectively; $p<0.0001$ for all fits) between the change in CM thresholds and the changes in $V_{\mathrm{U}}$ as a result of fluid in the middle-ear space (Fig. 6). That is, for all frequency ranges, as more fluid was added to the middle ear, the CM thresholds increased and the umbo velocity decreased. The linear regression produces an equation of the form $f=y_{0}+a(x)$, where $f$ is the change in CM threshold (i.e., the CHL experienced at the inner ear), $\mathrm{x}$ is the change in $V_{\mathrm{U}}$ (i.e., the umbo velocity estimate of the CHL), $y_{0}$ is the intercept, and $a$ the slope relating the change in $\mathrm{CM}$ threshold to the change in $V_{\mathrm{U}}$. The fitted parameters were $y_{0}=1.09 \mathrm{~dB}$ and $a=1.0 \mathrm{~dB} / \mathrm{dB}$ for the low frequency range, $2.35 \mathrm{~dB}$ and $1.04 \mathrm{~dB} / \mathrm{dB}$ for the medium frequency range and $3.1 \mathrm{~dB}$ and $1.02 \mathrm{~dB} / \mathrm{dB}$ for the high frequency range. A standard statistical bootstrap analysis (1,000 replications) of the regression data for each of the three frequency ranges revealed that the slope of the function relating CM threshold change to umbo velocity change was not significantly different than $1.0 \mathrm{~dB} / \mathrm{dB}$ (i.e., a slope of $1.0 \mathrm{~dB} / \mathrm{dB}$ fell within the bootstrap-derived $95 \%$ confidence intervals for slopes of the fits for the three frequency ranges). Thus, for all frequency ranges tested, MEEinduced increases in the CHL at the inner ear revealed by the $\mathrm{CM}$ were generally matched by the same reductions in umbo velocity.

When considered across all middle-ear fluid levels, the data reveal that the incremental reductions in $V_{\mathrm{U}}$ due to an MEE mostly explain the incremental increases in CHL as measured by CMs at the level of the inner ear. However, a closer inspection of the results indicate that there was generally a larger attenuation in CM thresholds as expressed in units of decibel SPL than the decibel amounts of attenuation in $V_{\mathrm{U}}$, particularly for fluid volumes that resulted in MEE-induced CHLs (based on CM thresholds) of $10 \mathrm{~dB}$ or greater. To show this more clearly, the CM threshold vs. umbo velocity data were compared over two different ranges, one corresponding to MEEinduced CM threshold increases of $10 \mathrm{~dB}$ or less (small CHL) and one corresponding to CM threshold increases greater than $10 \mathrm{~dB}$ (large CHL). For the high-frequency range (Fig. 6C), the change in $\mathrm{CM}$ thresholds for MEEs producing small CHLs (10 dB or less) was not significantly different (paired $t_{114}=-0.93$, $p<0.36$ ) than the corresponding change in $V_{\mathrm{U}}$, averaging $-0.54 \mathrm{~dB}(\mathrm{SD}, \pm 6.3 \mathrm{~dB})$. However, for MEEs producing large CHLs $(>10 \mathrm{~dB})$, the change in CM thresholds was significantly larger (paired $t_{129}=$ 6.67, $p<0.0001$ ) than the corresponding change in $V_{\mathrm{U}}$, 

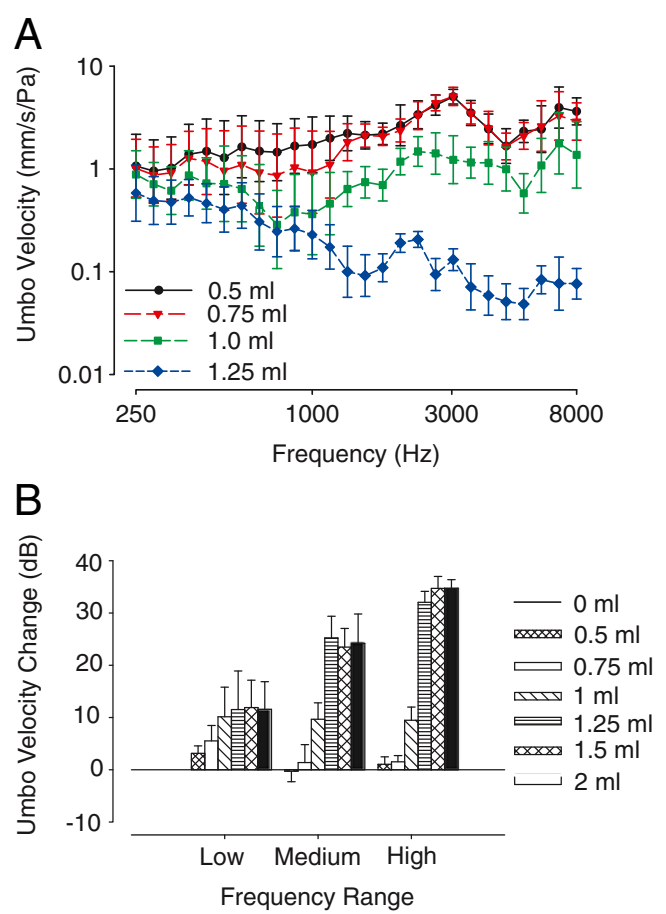

FIG. 5. Umbo velocity is reduced with increasing volume of middle-ear fluid. A Across-animal ( $n=5$ ears) mean umbo velocity transfer function (millimeter per second er Pascal) as a function of frequency; error bars indicate $\pm 1 \mathrm{SD}$. As the volume of middle-ear fluid was increased from $0.5 \mathrm{~mL}$, the mobility of the TM was reduced as indicated by a reduction in TM velocity. B The data in panel A were further analyzed by computing the MEE-induced change in umbo velocities relative to the baseline with no middle-ear fluid in three frequency ranges: low $(0.25-1 \mathrm{kHz})$, medium $(1-3 \mathrm{kHz})$, and high $(3-8 \mathrm{kHz})$. For low-frequency sound stimuli, umbo velocity decreased with the addition of $0.5 \mathrm{~mL}$ of fluid in the middle ear. Additional umbo velocity decreases occurred with $0.75 \mathrm{~mL}$ and $1.0 \mathrm{~mL}$ of instilled fluid. Maximum changes in umbo velocities for low frequencies $(\sim 10 \mathrm{~dB})$ were reached by $1.0 \mathrm{~mL}$ of middle-ear fluid. For medium- and high-frequency sound stimuli, decreases in umbo velocity initially occurred at $1.0 \mathrm{~mL}$ of fluid in the middle-ear space. Additional decreases in umbo velocity occurred with $1.25 \mathrm{~mL}$ of fluid in the middle ear, with no significant difference in umbo velocity decrease occurring above $1.25 \mathrm{~mL}$ of fluid. Maximum decrease in umbo velocity for medium- and high-frequency sounds was $\sim 25$ and $\sim 35 \mathrm{~dB}$, respectively. Baseline measurements are not shown as they are not significantly different from $0.5-\mathrm{mL}$ fluid instillation values for umbo velocity measurements.

averaging $6.65 \mathrm{~dB}(\mathrm{SD}, \pm 11.4 \mathrm{~dB})$ larger. Similarly, for the medium frequencies (Fig. 6B), changes in $\mathrm{CM}$ thresholds for MEEs producing large CHLs were $5.94 \mathrm{~dB}( \pm 8.17 \mathrm{~dB})$ larger than the change in $V_{\mathrm{U}}$, which also reached significance (paired $t_{148}=8.84, p<$ $0.0001)$; the differences were not significant for MEEs producing small CHL (paired $t_{131}=-1.67, p<0.1$ ). Finally, for the low-frequency range (Fig. 6A), changes in CM threshold were significantly larger (paired $t_{130}=$ $10.4, p<0.0001)$ by $7.18 \mathrm{~dB}( \pm 7.9 \mathrm{~dB})$ than the changes in $V_{\mathrm{U}}$ for MEEs producing large CHL; for MEEs producing small CHL the differences were also
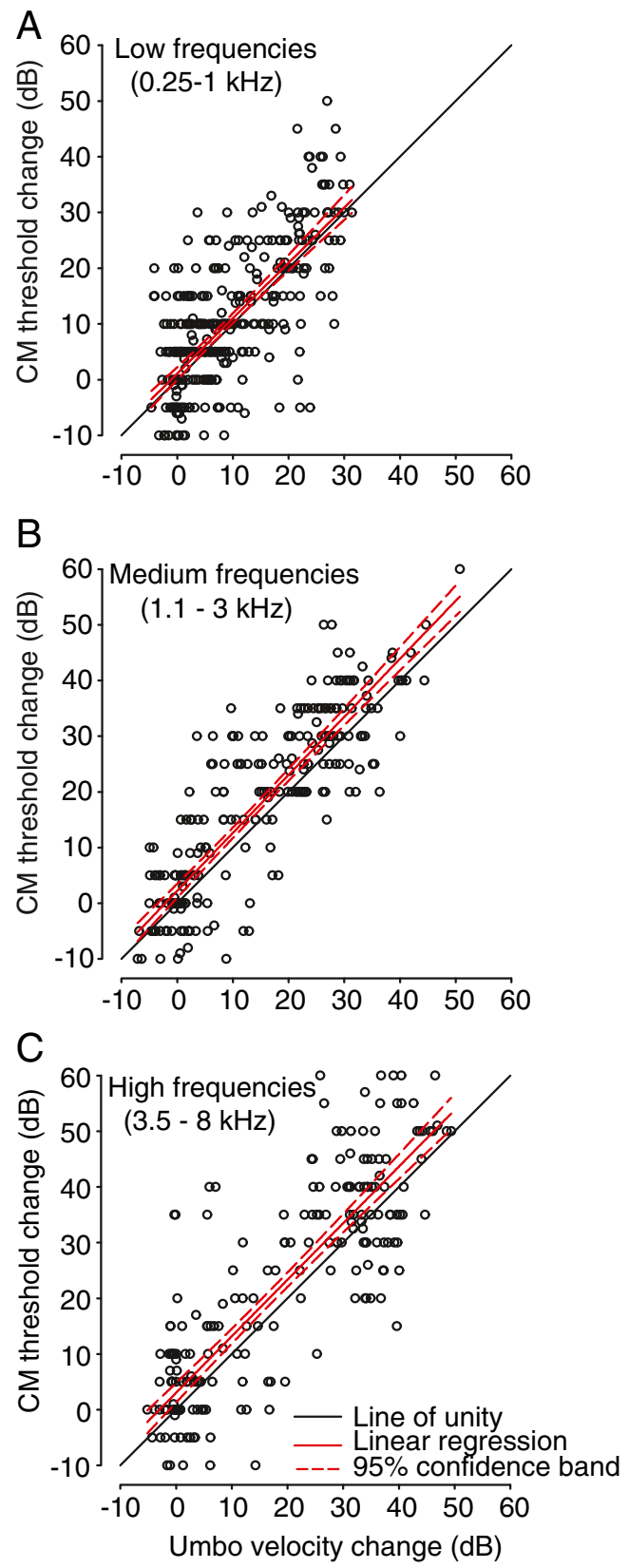

FIG. 6. Correlation between middle-ear fluid induced changes in CM thresholds and umbo velocities for the $\mathbf{A}$ low-, B medium-, and $\mathbf{C}$ high-frequency ranges. Positive values indicate increased $\mathrm{CM}$ thresholds (i.e., increased $\mathrm{CHL}$ ) and decreased umbo velocities, respectively. For each frequency range, with increases in the volume of fluid in the middle ear, there was a significant positive correlation between the CM threshold increases and umbo velocity decreases (see main text). The completely filled middle ear caused a frequencydependent 20-40-dB increase in CM thresholds (Fig. 2), but only a 15-35-dB attenuation in umbo velocities (Fig. 5). In each panel, the solid black line indicates the unity line (slope $=1.0 \mathrm{~dB} / \mathrm{dB}$ ), the thick red line indicates the linear regression function relating changes in CM thresholds to changes in umbo velocity, and the dashed lines indicate the $95 \%$ confidence interval for the regression function.

significant (paired $t_{255}=-2.48, p<0.01$ ), but the size of the difference was small, $-0.99 \mathrm{~dB}( \pm 6.1 \mathrm{~dB})$. These 
results demonstrate that MEE-induced reductions in TM movement, at least as measured by $V_{\mathrm{U}}$, explain most, but not the total, amount of sound attenuation at the level of the inner ear.

\section{DISCUSSION}

In this study, we used a combination of tympanometry, umbo velocity, and CM measurements to determine how the middle-ear system (consisting of the TM, ossicular chain, and oval window) transmits sound input to the inner ear in the presence of a MEEinduced CHL. By combining these three techniques, we aimed to gain a more comprehensive understanding of how MEE-induced CHL affects the different aspects of sound transmission: from airborne sound waves, to movement of the TM, through the middle ear and ultimately to the inner ear. Additionally, these experiments explored the clinically relevant question of how much middle-ear fluid is necessary to cause a clinically detectable CHL.

Tympanometry using higher probe frequencies is more sensitive to MEE than clinic-standard $226 \mathrm{~Hz}$ tympanometry-a case for multi-frequency tympanometry

Tympanometry is designed to measure the movement of the TM in response to sound stimulation and is frequently used clinically to assess for the presence of an MEE. Normally, without fluid in the middle ear, the TM is able to move freely in response to sound pressure fluctuations. Tympanometry performed in the clinic is done using a probe frequency of $226 \mathrm{~Hz}$, a frequency that is optimized for adult-sized middle-ear spaces. However, previous work, along with the current study, indicates that $226 \mathrm{~Hz}$ is often not sufficiently sensitive enough to rule out the presence of MEE (Orchik et al. 1978; Wiederhold et al. 1980; Margolis et al. 1995; Rogers et al. 2010), especially in infants that have smaller middle-ear volumes than adults (Paradise et al. 1976; Calandruccio et al. 2006).

Margolis et al. (1995) found that, similar to our current study, a $226-\mathrm{Hz}$ probe frequency is not sensitive enough to detect $0.5 \mathrm{~mL}$ of fluid in the middle ear of adult chinchillas. With $1.0 \mathrm{~mL}$ and $1.5 \mathrm{~mL}$ of saline in the middle space, which they reported as corresponding to $56 \%$ and $83 \%$ of the average volume of the bullae, respectively, Margolis et al. (1995) observed flat, type B tympanograms using a $226-\mathrm{Hz}$ probe frequency. However, we typically continued to observe type A tympanograms at $226 \mathrm{~Hz}$ with both $1.0 \mathrm{~mL}$ and $1.5 \mathrm{~mL}$ of silicone oil in the middle ear. Type B tympanograms were only observed if a higher probe frequency (i.e., $1,400 \mathrm{~Hz})$ was used. This indicates that the clinic- standard 226- $\mathrm{Hz}$ probe frequency is not consistently able to detect the presence of fluid in the middle ear, even when a large area of the TM is covered by fluid.

Chinchillas have a middle-ear space volume directly behind the TM that is comparable to the volume of the human tympanic cavity directly behind the TM $\left(\sim 1.8 \mathrm{~cm}^{3}\right.$; Vrettakos et al. 1988; Margolis et al. 1995;), although the volume of the entire middle-ear air space in humans is larger than that of the chinchilla $\left(\sim 6.5 \mathrm{~cm}^{3}\right.$; Zwislocki 1962; Molvaer et al. 1978) suggesting that the effects of fluid volume on tympanometry found in chinchillas could also translate to human subjects. It is important to note, however, that chinchillas have a hypermobile TM and that opening the middle-ear cavity can cause increases in middle-ear movement (Rosowski et al. 2006). This is in contrast to human subjects, where opening the middle ear has minimal effects on TM mobility (Zwislocki 1962).

A $226-\mathrm{Hz}$ probe tone frequency is often favored clinically due to the decrease in static immittance (immittance of the middle ear at rest) errors that occur at this frequency (Van Camp et al. 1986). Static immittance measurements are not useful as a diagnostic tool on their own, but can provide additional information about middle-ear pathologies (i.e., otosclerosis, cholesteatoma) when combined with other audiometric tests (Jerger et al. 1974; Hall and Chandler 1994). Multi-frequency tympanometry (MFT) uses a range of higher probe frequencies to test the admittance magnitude of the TM and is more sensitive to the presence of MEE (Williams et al. 1995; Purdy and Williams 2000; Lai et al. 2008; Margolis et al. 1995). In chinchillas, Margolis et al. (1998) demonstrated that MFT combined with a $226-\mathrm{Hz}$ tympanometry is more effective in diagnosing middle-ear pathologies than either technique alone, and that some pathologies that could not be detected with a 226-Hz tympanometry produced abnormal MFT results. Although chinchillas have a larger middle ear admittance than humans (most likely due to small ossicles and a thin hypermobile TM), MFT patterns in chinchillas are qualitatively similar to MFT patterns in humans (Margolis et al. 1995). The current study found similar results using MFT in that MFT was more accurate in confirming the presence of fluid in the middle-ear space. However, MFT can be difficult to interpret due to multiple admittance peak measurements which do not adhere to the traditional Jerger (1970) method of classification. Using higher probe frequencies may be better for detecting the presence of OME in children (Marchant et al. 1986), as a $226-\mathrm{Hz}$ probe tone is more suitable for adults as opposed to infants and children (Bennett and Weatherby 1982).

Previous studies in both children and adults have used wideband acoustic transfer functions (Ellison et 
al. 2012; Keefe and Simmons 2003) and energy reflectance measurements (Nakajima et al. 2012; Feeney et al. 2003) as methods to detect the presence of CHL or other middle ear abnormalities. Absorbance data from wideband acoustic transfer function measurements were reduced in the affected ears of children with a middle-ear effusion, and served as a better predictor of conductive hearing loss than 226-Hz tympanometry (Ellison et al. 2012). Additionally, using multiple testing variables (admittance, absorbance, reflectance, etc.) together was more effective at diagnosing middle-ear pathologies than tympanometry alone (Ellison et al. 2012; Piskorski et al. 1999; Keefe and Simmons 2003). However, these types of more advanced diagnostic methods (wideband acoustic transfer functions and energy reflectance) are not routinely used in the clinic to diagnose the presence of CHL.

Although tympanometry alone cannot provide much data about the movement of the ossicular chain, it can supply information about the physical properties of the TM, as well as middle-ear compliance and pressure. Dai and Gan (2008) studied TM properties in guinea pigs with MEEs caused by an injection of lipopolysaccharide (LPS) into the middle ear. Injection of LPS into the middle-ear space causes inflammation and subsequent effusion (Ohashi et al. 1991). All LPSinjected ears had a middle-ear space that was at least half-filled with fluid. Using multi-frequency tympanometry, they found that the mean values of compliance in the middle ear were reduced in animals with LPS-injected ears relative to normal. A reduced compliance in LPS-injected ears indicates a reduction in the movement of the TM. If TM mobility is compromised, this could introduce an attenuation of sound transmission through the middle ear resulting in a CHL.

\section{Umbo velocity changes resulting from MEEs} are volume and frequency-dependent

We found here that the velocity of the TM movement as measured by umbo velocity in response to sound is compromised in experimentally induced MEEs. Ravicz et al. (2004) also used the umbo velocity transfer function $\left(H_{\mathrm{UV}}\right)$ as a measurement of $\mathrm{CHL}$ in a cadaveric human temporal bone model of OME to study the etiology of MEE-related CHL. In that study, Ravicz et al. (2004) discovered that the effects of middle-ear fluid (saline, in this case) on $H_{\mathrm{UV}}$ are frequency-dependent. At low frequencies (100$200 \mathrm{~Hz}$ ), reduction in $H_{\mathrm{UV}}$ is presumably caused by a reduction in middle-ear air volume due to the amount of fluid in the middle-ear space. Additionally, reduction in $H_{\mathrm{UV}}$ at low frequencies was not dependent on the viscosity of the fluid. At frequencies $>1 \mathrm{kHz}$, changes in $H_{\mathrm{UV}}$ were associated with the area of the TM in contact with fluid, even when the volume of the effusion was held constant and the temporal bone was reoriented to independently assess the effect of TM area-fluid contact on $H_{\mathrm{UV}}$.

Guan and Gan (2011) performed a similar experiment in guinea pigs, looking at the movement of the TM (at the umbo), incus, and round window when the middle ear was filled with saline. Like Ravicz et al. (2004), they found that middle-ear fluid reduced TM movement more at high frequencies compared to low frequencies. Presumably, at higher frequencies, the movement of the TM becomes more mass-dominated, so increasing the amount of fluid that is contacting the TM results in an effective increase of the TM mass, regardless of the viscosity of the fluid (Ravicz et al. 2004). Our results are wholly consistent with previous studies (Ravicz et al. 2004; Gan et al. 2006; Dai and Gan 2008; Guan and Gan 2011; Thornton et al. 2012), as we also saw a greater reduction in umbo velocity at higher frequencies $(3-8 \mathrm{kHz})$ than at lower frequencies (Fig. 5).

In addition to being frequency-dependent, changes in umbo velocity were also dependent on the volume of fluid present in the middle-ear space. For middle (1-3 kHz) and high (3-8 kHz) frequency sounds, we observed a significant change in umbo velocity after $1.25 \mathrm{~mL}$ of fluid was instilled into the middle-ear space. However, after adding additional fluid, the umbo velocity was not significantly different from the umbo velocity with $1.25 \mathrm{~mL}$ of fluid in the ear. Again, this is most likely due to the fact that at middle and high frequencies, TM movement is more reliant on how much of the TM is contacted by fluid; thus, increased fluid volume in the middle ear results in an increase of fluid that is available to contact the TM. The present results in the chinchilla should be tempered by the fact that the middle ear was vented by a 2-3-mm diameter hole in the superior part of the bulla. Rosowski et al. (2006) has shown that when the bullae of chinchilla are vented with a similar size hole the compliance dominance of the middle ear shifts from frequencies less than $\sim 1 \mathrm{kHz}$ (in a sealed bulla) to frequencies less than $\sim 100 \mathrm{~Hz}$.

\section{CM thresholds increase in a sound frequency and TM area-contact-dependent manner}

The cochlear microphonic (CM) measurement represents the summed activity of the outer hair cells and is used as a measure of peripheral auditory system activity (Wever and Lawrence 1954; Dallos 1973). CM recordings have been used previously to gather information about how CHL (including MEE-induced CHL) alters the acoustic cues to sound location 
(Knudsen et al. 1984; Hartley and Moore 2003; Lupo et al. 2011; Thornton et al. 2012). Additionally, CM measurements provide information about the efficiency of the middle ear in transferring sound energy from the external ear canal, through the middle ear, and ultimately to the inner ear. By measuring the inputs to the middle-ear system (tympanometry and $V_{\mathrm{U}}$ ) and comparing these to CM thresholds (measurement of inner ear activity), we are able to establish a more comprehensive assessment of the physiology of the middle ear as a result of MEE-induced CHL.

Similar to $V_{\mathrm{U}}$ attenuation, fluid volumes of $1.0 \mathrm{~mL}$ in the middle ear were sufficient to cause CM threshold elevations in all frequencies tested. Greater increases in $\mathrm{CM}$ thresholds were seen at higher stimulus frequencies $(3-8 \mathrm{kHz})$. The largest increases in CM thresholds were seen when the TM was fully covered by fluid. Similar instances of MEEinduced CM threshold elevation were observed in previous studies (Hartley and Moore 2003; Petrova et al. 2006; Guan and Gan 2011). Guan and Gan (2011) used auditory brainstem response (ABR) thresholds to study the peripheral auditory system response to MEEinduced CHL in guinea pigs. They found that ABR thresholds increased significantly across most frequencies with $1.0 \mathrm{~mL}$ of fluid in the middle ear and increased by even larger amounts when there was $2.0 \mathrm{~mL}$ of fluid present in the middle ear. Furthermore, they observed that round window membrane movement latencies were increased at all frequencies in the presence of middle-ear fluid, suggesting that middle-ear fluid also affects the temporal properties of the transmission of sound energy from the middle ear to the inner ear. Similar findings have been reported by Ravicz et al. (2004) and Thornton et al. (2012) in the temporal bone and animal models of MEE, respectively.

We demonstrated here that the effect of an MEEinduced CHL at the level of the inner ear (as measured by cochlear microphonics) can be mostly predicted by the reduced movement of the tympanic membrane (as measured by umbo velocity). However, for MEEs that produced CHLs of $10 \mathrm{~dB}$ or greater, reductions in umbo velocity were $\sim 6 \mathrm{~dB}$ less than the CHL measured via CM threshold elevation across all frequency ranges. Thus, additional mechanisms more proximal than the TM must likely cause the additional $6 \mathrm{~dB}$ of CHL.

The notion that reductions in umbo velocity cannot explain all of the CHL at the inner ear is not without precedent. Previous studies have found similar results that while tympanic membrane mobility is decreased in the presence of an MEE-induced CHL, the level of attenuation measured objectively at the inner ear is greater. In our measurements, we found that there was on average an approximately 6-dB difference in attenuation between umbo velocity and CM measurements (Fig. 6). Guan and Gan (2011) compared the effects of MEE at the level of the middle and inner ears by measuring ossicular mobility and ABR thresholds, respectively. At higher frequencies $(10-20 \mathrm{kHz})$, differences between TM mobility measurements and ABR thresholds were also $\sim 6 \mathrm{~dB}$. Moreover, Turcanu et al. (2009) also found a similar $\sim 6-\mathrm{dB}$ difference between umbo velocity measurements and compound action potential responses in their study of middle-ear effusion. However, Qin et al. (2010) in mouse reported larger reductions in umbo velocity due to saline-filled bullae than the increases in ABR thresholds. Thus, reductions in umbo velocity and TM mobility do not always explain all of the CHL experienced at the inner ear due to MEE. For future studies, it would be interesting to evaluate the receiver operating characteristic (ROC) curve for each test (CM thresholds, umbo velocity, tympanometry measurements, etc.) to determine if the ROC curve analysis would be able to predict the magnitude of CHL. Previous studies have used areas under the ROC curve to predict CHL and found that 226- $\mathrm{Hz}$ tympanometry was less sensitive than wideband acoustic transfer functions in predicting CHL (Keefe and Simmons 2003).

The presence of an MEE is not necessarily indicative of the presence of a $\mathrm{CHL}$-implications for long-term hearing impairments due to chronic $\mathrm{CHL}$

Although most children experience multiple bouts of OME during early childhood, it is less clear whether OME typically presents with a corresponding CHL. This distinction is critical, as the mere presence of OME alone without associated CHL would not be expected to result in long-term hearing deficits (Roberts et al. 2004). However, OME that has been clinically documented to coincide with a corresponding CHL has been shown to result in altered auditory physiology and auditory behavior in a majority of cases (for review, see Whitton and Polley 2011); children with these conditions have a high likelihood of developing persistent hearing deficits. To arrive at this conclusion, Whitton and Polley (2011) performed a metaanalysis of longitudinal, retrospective, and randomized control studies on the presence of CHL, otitis media and hearing abilities, and found that only $53 \%$ of study samples exhibited long-term auditory processing difficulties as a result of otitis media. However, this result contrasts sharply with the $89 \%$ of study samples that reported auditory processing deficits as a result of otitis media occurring jointly with CHL. Thus, OME by itself is 
not predictive of long-term and persistent hearing deficits, whereas objective measurement of an associated CHL is predictive. Why don't all OME cases lead to CHL? The present paper addresses this conundrum by exploring how much fluid is required to detect $\mathrm{OME}$ clinically and how much fluid (percent TM coverage) is needed to cause a mild-moderate CHL. We report here that only when the TM is almost completely covered with fluid due to an MEE that a mild-moderate CHL $(\sim 20-40 \mathrm{~dB})$ will be present. It is children presenting with these magnitudes of CHLs that require aggressive and closely monitored treatment, as the amelioration of the CHL is critical for normal auditory processing. On the other hand, clinically, OME might be present (as indicated by tympanometry and visual inspection), but the CHL could be negligible. Thus, children with chronic OME should ideally receive hearing tests to determine the presence and magnitude of an MEEinduced CHL (Roberts et al. 2004).

\section{ACKNOWLEDGMENTS}

This work was supported by the National Institutes of Deafness and Other Communicative Disorders (NIDCD) grant F31-DC011198, T32-NS007083, and T32-HD041697 to JLT and NIDCD R01-DC011555 to DJT. Support for the initial phases of this work was provided by the National Organization for Hearing Research (NOHR) Evie and Ron Krancer Grant in Auditory Science to DJT. We thank Drs. Eric Lupo and Kristin Uhler for their helpful comments about the experiments and manuscript and Kelsey Anbuhl and Alex Harbison for construction of Figure 1.

\section{REFERENCES}

Bennett MJ, Weatherby LA (1982) Newborn acoustic reflexes to noise and pure-tone signals. J Speech Hear Res 25:383-387

Bluestone CD, KueIn JO (eds) (1995) Definitions, terminology, and classification. In: Otitis media in infants and children, 2nd edn. W.B. Saunders Co., Philadelphia p 1-3

Bluestone CD, Beery QC, Paradise JL (1973) Audiometry and tympanometry in relation to middle ear effusions in children. Laryngoscope 83:594-604

BRown DT, MARSh RR, PotsIC WP (1983) Hearing loss induced by viscous fluids in the middle ear. Int J Pediatr Otorhinolaryngol 5:39-46

Browning GC, Granich M (1978) Surgical anatomy of the temporal bone in the chinchilla. Ann Otol Rhinol Laryngol 87:875-882

Calandruccio L, Fitzgerald TS, Prieve BA (2006) Normative multifrequency tympanometry in infants and toddlers. J Am Acad Audiol 17:470-480

DAI C, GAN RZ (2008) Change of middle ear transfer function in otitis media with effusion model of guinea pigs. 243:78-86

Dallos P (1973) The auditory periphery: biophysics and physiology. Academic Press, New York

Ellison JC, Gorga M, Cohn E, Fitzpatrick D, Sanford CA, Keefe DH (2012) Wideband acoustic transfer functions predict middle-ear effusion. Laryngoscope 122:887-894
Feeney MP, Grant IL, Marryott LP (2003) Wideband energy reflectance measurements in adults with middle-ear disorders. J Speech Lang Hear Res 46:901-911

GAN RZ, DAI C, WoOD MW (2006) Laser interferometry measurements of middle ear fluid and pressure effects on sound transmission. J Acoust Soc Am 120:3799-3810

Goodhill V, Holcomb AL (1958) The relation of auditory response to the viscosity of tympanic fluids. Acta Otolaryngol 49:38-46

Guan X, GAN RZ (2011) Effect of middle ear fluid on sound transmission and auditory brainstem response in guinea pigs. Hear Res 277:96-106

Hall JW, Chandler DW (1994) Clinical tympanometry. In: Katz J, Ruth R (eds) Handbook of clinical audiology, 4th edn. Williams and Wilkins, Baltimore

Hall JW, Derlacki EL (1986) Effect of conductive hearing loss and middle ear surgery on binaural hearing. Ann Otol Rhinol Laryngol 95:525-530

Hall JW, Grose JH, Dev MB, Ghiassi S (1998) The effect of masker interaural time delay on the masking level difference in children with history of normal hearing or history of otitis media with effusion. Ear Hear 19:220-229

Hartley DEH, MoOre DR (2003) Effects of conductive hearing loss on temporal aspects of sound transmission through the ear. Hear Res 177:53-60

HeffNer RS, HefFner HE (1991) Behavioral hearing range of the chinchilla. Hear Res 52:13-16

Himmelfarb MZ, Popelka GR, Shanon E (1979) Tympanometry in normal neonates. J Speech Hear Res 22:179-191

HogAN SC, MOORE DR (2003) Impaired binaural hearing in children produces by a threshold level of middle ear disease. J Assoc Res Otolaryngol 4(2):123-129

Holte L, Margolis RH, Cavanagh RM (1991) Developmental changes in multifrequency tympanograms. Audiology 30:1-24

JERGER JF (1970) Clinical experience with impedance audiometry. Arch Otolaryngol 92:311-324

Jerger J, Anthony L, Jerger S, Mauldin L (1974) Studies in impedance audiometry. 3. Middle ear disorders. Arch Otolaryngol 99:165-171

Jeselsohn Y, Freeman S, Segal N, Sohmer H (2005) Quantitative experimental assessment of the factors contributing to hearing loss in serous otitis media. Otol Neurotol 26:10111015

Joint Committee on Infant Hearing (2007) Position statement: principles and guidelines for early hearing detection and intervention programs. Pediatrics 120:898-921

KeEfe DH, Simmons JL (2003) Energy transmittance predicts conductive hearing loss in older children and adults. J Acoust Soc Am 114:3217-3238

Keith RW (1975) Middle ear function in neonates. Arch Otolaryngol 101:376-379

Knudsen EI, Esterly SD, Knudsen PF (1984) Monaural occlusion alters sound localization during a sensitive period in the barn owl. J Neurosci 4:1001-1011

Кокко E (1974) Chronic secretory otitis media in children: a clinical study. Acta Otolaryngol Suppl 327:1-44

Lai D, Li W, Xian J, Liu S (2008) Multifrequency tympanometry in adults with otitis media with effusion. Eur Arch Otorhinolaryngol 265:1021-1025

Lupo JE, Koka K, Thornton JL, Tollin DJ (2011) The effects of experimentally induced conductive hearing loss on spectral and temporal aspects of sound transmission through the ear. Hear Res 272:30-41

Marchant CD, McMillan PM, Shurin PA, Johnson CE, Turczyk VA, Feinstein JC, Panek DM (1986) Diagnosis of otitis media in early infancy by tympanometry and ipsilateral acoustic reflex thresholds. J Pediatr 109:590-595 
Margolis RH, Popelka GR (1975) Static and dynamic acoustic impedance measurements in infant ears. J Speech Hear Res 18:435-453

Margolis RH, Schachem PL, Hunter Ll, Sutherland C (1995) Multifrequency tympanometry in chinchillas. Audiology 34:232-247

Margolis RH, Schachern PA, Fulton S (1998) Multifrequency tympanometry and histopathology in chinchillas with experimentally-produced middle-ear pathologies. Acta Otolaryngol 118:216-225

Marsh RR, Baranak CC, Potsic WP (1985) Hearing loss and viscoelasticity of middle ear fluid. Int J Pediatr Otorhinolaryngol 9:115-20

MilleR JD (1970) Audibility curve of the chinchilla. J Acoust Soc Am 48:513-523

Molvaer O, Vallersnes F, Kringelbotn M (1978) The size of the middle and the mastoid air cell. Acta Otolaryngol 111:10-16

Moore DR, Hutchings ME, MeYer SE (1991) Binaural masking level differences in children with a history of otitis media. Audiology 30:91-101

Moore DR, Hartley DE, Hogan SC (2003) Effects of otitis media with effusion (OME) on central auditory function. Int J Pediatr Otorhinolaryngol 67(Suppl 1):S63-S67

Nakajima HH, Pisano DV, Roosli C, Hamade MA, Merchant GR, Mahfoud L, Halpin CF, Rosowski JJ, Merchant SN (2012) Comparison of ear-canal reflectance and umbo velocity in patients with conductive hearing loss: a preliminary study. Ear Hear 33:35-43

Niemiec AJ, Yost WA, SHofner WP (1992) Behavioral measures of the frequency selectivity in the chinchilla. J Acoust Soc Am 92:26362649

Ohashi Y, Nakai Y, Esaki Y, Ohno Y, Sugiura Y, Okamoto H (1991) Experimental otitis media with effusion induced by lipopolysaccharide from Klebsiella pneumoniae. Mucociliary pathology of the eustachian tube. Acta Otolaryngol Suppl 486:105-115

Orchik DJ, Dunn JW, McNutT L (1978) Tympanometry as a predictor of middle ear effusion. Arch Otolaryngol 104:4-6

Paradise JL, Smith CG, Bluestone CD (1976) Tympanometric detection of middle ear effusion in infants and young children. Pediatrics 58:198-210

Paradise JL, Rockette HE, Colburn DK et al (1997) Otitis media in 2253 Pittsburgh area infants: prevalence and risk factors during the first two years of life. Pediatrics 99:318-333

Petrova P, Freeman S, Sohmer H (2006) The effects of positive and negative middle ear pressures on auditory thresholds. Otol Neurotol 27:734-738

Pillsbury HC, Grose JH, Hall JW (1991) Otitis media with effusion in children: binaural hearing before and after corrective surgery. Arch Otolaryngol Head Neck Surg 117:718-723
Piskorski P, Keefe DH, Simmons JL, Gorga MP (1999) Prediction of conductive hearing loss based on acoustic ear-canal response using a multivariate clinical decision theory. J Acoust Soc Am 105:1749-1764

Purdy SC, Williams MJ (2000) High frequency tympanometry: a valid and reliable immittance test protocol for young infants? N Z Audiol Soc Bull 10:9-24

Qin Z, Wood M, Rosowski JJ (2010) Measurement of conductive hearing loss in mice. Hear Res 263:93-103

Ravicz ME, Rosowski JJ, Merchant SN (2004) Mechanisms of hearing loss resulting from middle-ear fluid. Hear Res 195:103-130

Roberts JE, Rosenfeld RM, Zeisel SA (2004) Otitis media and speech and language: a meta-analysis of prospective studies. Pediatrics 113:E238-E248

Rogers DJ, Boseley ME, Adams MT, Makowski RL, Hohman MH (2010) Prospective comparison of handheld pneumatic otoscopy, binocular microscopy, and tympanometry in identifying middle ear effusions in children. Int J Pediatr Otorhinolaryngol 74:1140 1143

Rosowski JJ, Ravicz ME, Songer JE (2006) Structures that contribute to middle-ear admittance in chinchilla. J Comp Physiol A Neuroethol Sens Neural Behav Physiol 192:1287-311

Thornton JL, Chevaluier KM, Koka K, Lupo JE, Tollin DJ (2012) The conductive hearing loss due to an experimentally-induced middle ear effusion alters the interaural level and time differences cues to location. J Assoc Res Otolaryngol 13:641654

Turcanu D, Dalhoff E, Muller M, Zenner HP, Gummer AW (2009) Accuracy of velocity distortion product otoacoustic emissions for estimating mechanically based hearing loss. Hear Res 251:1728

Van Camp KJ, Margolis RH, Wilson RH, Creten WL, Shanks Je (1986) Principles of tympanometry. ASHA Press, Rockville

VRettakos PA, Dear SP, Saunders JC (1988) Middle ear structure in the chinchilla: a qualitative study. Am J Otolaryngol 9:58-67

Wever EG, Lawrence M (1954) Physiological acoustics. Princeton University Press, Princeton

Whitton JP, Polley DB (2011) Evaluating the perceptual and pathophysiological consequences of auditory deprivation in early postnatal life: a comparison of basic and clinical studies. J Assoc Res Otol 12:535-546

Wiederhold ML, Zajtchuk JT, VAP JG, PagGi RE (1980) Hearing loss in relation to physical properties of middle ear effusions. Ann Otol Rhinol Laryngol 89:185-189

Williams MJ, Purdy SC, Barber C (1995) High frequency probe tone tympanometry in infants with middle ear effusions. Austr J Otolaryngol 2:169-173

ZwIsLocki J (1962) Analysis of the middle-ear function. I. Input impedance. J Acoust Soc Am 34:1514-1523 